\title{
Real-space quadrature: a convenient, efficient representation for multipole expansions
}

\author{
David M. Rogers \\ University of South Florida, 4202 E. Fowler Ave., CHE 205, Tampa, FL 3362d*
}

(Dated: November 4, 2014)

\begin{abstract}
Multipolar expansions are a foundational tool for describing basis functions in quantum mechanics, many-body polarization, and other distributions on the unit sphere. Progress on these topics is often held back by complicated and competing formulas for calculating and using spherical harmonics. We present a complete representation for supersymmetric 3D tensors that replaces spherical harmonic basis functions by a dramatically simpler set of weights associated to discrete points in $3 \mathrm{D}$ space. This representation is shown to be space optimal. It reduces tensor contraction and the spherical harmonic decomposition of Poisson's operator to pairwise summations over the point set. Moreover, multiplication of spherical harmonic basis functions translates to a direct product in this representation.
\end{abstract}

\section{INTRODUCTION}

Multipole expansions are used throughout QM, advanced MM, and large-scale electrostatics calculations. They appear as derivatives of $r^{-1}$ in the Green's function solution to Poisson's equation. Applications include the computation of angular interaction matrix elements (themselves based on spherical harmonic functions) in quantum codes, 1 3 fast $O(N)$ implicit solvent calculations in molecular dynamics 44 6] and fluid flows, [7, 8] describing molecular polarization 913 , and boundary representations of dielectric polarization in molecular cavities. 14 17

Despite their wide-ranging applications, actual use of these expansions is hindered by the tenuous connections between spectral (spherical harmonic) and real-space (Cartesian) formalisms. [10] Essentially all serious applications make use of manually tabulated Cartesian forms, often implemented by hand. [7, 11, 14, 18 21] This strategy is error-prone, time-consuming and useful only for low-order modes. This leads, for example, to widely-used and highly-regarded quantum chemistry codes that only support angular basis functions up to order 6, 222] and simulation methods and codes for which even implementations at the dipole 23 or quadrupole 24, 25] level are great advancements. In addition, there is a gap in understanding translations from harmonics to representations relying completely on point-charges, 13 . or on explicit supersymmetric tensors. 24, 26] The straightforward route for a point-charge representation of multipoles up to order $p-1$ requires $2^{p}$ charges, 2, 18, while a tensor representation requires $3^{p}$ tensor elements. In this work we show space-optimal representations for point-charges and Cartesian tensors, and provide simple translations between these three formalisms.

The ubiquitous success of spherical harmonics stems from the separation of source and destination points $(x$ and $y$ ) in the expansion of the potential function,

$$
\frac{1}{|y-x|}=\sum_{l=0}^{\infty} \sum_{m=-l}^{l} O_{l m}(y) M_{l m}(x), \quad|y|<|x|
$$

where $x$ and $y$ are three-dimensional vectors, and

$$
\begin{aligned}
O_{l m}(x) & =|x|^{l}(l+|m|) ! P_{l m}\left(\cos \theta_{x}\right) e^{-i m \phi_{x}} \\
M_{l m}(x) & =|x|^{-l-1}(l-|m|) !^{-1} P_{l m}\left(\cos \theta_{x}\right) e^{i m \phi_{x}}
\end{aligned}
$$

This simplified form is due to Ref. [27]. Here, $i$, is the unit imaginary number and $P_{l m}$ are the associated Legendre polynomials.

We contend that for almost all real applications, this decomposition goes one step too far. Rather, it is preferable to stop at the unadorned Legendre polynomials,

$$
P_{l}(\hat{x} \cdot \hat{y})=\sum_{m=-l}^{l} O_{l m}(\hat{x}) M_{l m}(\hat{y})
$$

where $\hat{x}$ represents a three-dimensional unit vector, $|\hat{x}|=$ 1. The $P_{l}$ are polynomials of degree $l$ in the vector innerproduct, $\hat{x} \cdot \hat{y}$, and are obviously symmetric to interchanging $x$ and $y$. No angles need to be defined or used.

In preference to the pair, $O$ and $M$, we then use scaled Legendre polynomials,

$$
L_{n}(x, y) \equiv \frac{|x|^{n}}{|y|^{n+1}} P_{n}(\hat{x} \cdot \hat{y}) .
$$

When scaled by $|y|^{2 n+1}$, the $L_{n}$ are polynomials in the inner products, $x \cdot x, y \cdot y$, and $x \cdot y$, symmetric to interchange of $x$ and $y$. They can be generated by a two-term recurrence [since $L_{n}(x, y)=F_{n}(x, y ;-1)$,

$$
\begin{aligned}
F_{n}(x, y ; \alpha) & \equiv\left(-x \cdot \partial_{y}\right)^{n}|y|^{\alpha} / n ! \\
& =|y|^{\alpha}, \quad n=0 \\
& =-\alpha \frac{x \cdot y}{y \cdot y}|y|^{\alpha}, \quad n=1 \\
& =\frac{2 n-2-\alpha}{n} \frac{x \cdot y}{y \cdot y} F_{n-1}(x, y ; \alpha) \\
& +\frac{\alpha+2-n}{n} \frac{x \cdot x}{y \cdot y} F_{n-2}(x, y ; \alpha) .
\end{aligned}
$$




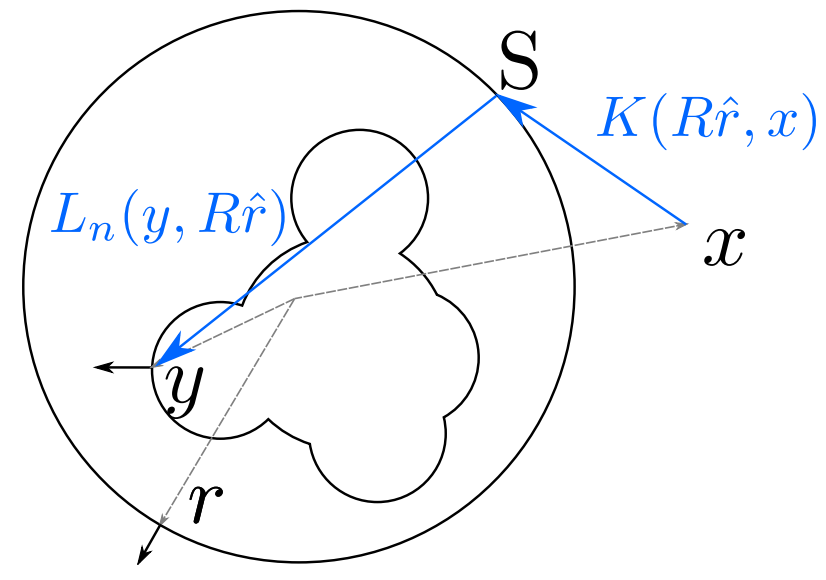

FIG. 1. Illustration of quadrature representation of the multipole expansion. The source at position $y$ is transferred to the quadrature points, $R \hat{r}_{i}$ (using Eq. 12 ). Its potential inside the sphere is calculated from those effective charges (Eq. 9). Normal vector directions (pointing out of the sphere) are defined for boundary integrals.

They express successive derivatives of $|y|^{-1}$, so that

$$
\frac{1}{|x-y|}=e^{-x \cdot \partial_{y}}|y|^{-1}=\sum_{n=0}^{\infty} L_{n}(x, y) .
$$

\section{A. Definition of the Quadrature Representation}

Identities previously phrased in terms of harmonics find a much simpler expression using the reproducing kernel (Fig. 11, 28]

$$
K(x, y)=\sum_{n=0}^{p-1} \frac{2 n+1}{4 \pi} L_{n}(x, y) .
$$

Its status as an effective identity kernel can be shown using the orthogonality of the Legendre polynomials on the surface of the unit sphere $(S), 28$.

$$
\int_{S} P_{n}(\hat{x} \cdot \hat{r}) P_{m}(\hat{r} \cdot \hat{y}) d^{2} \hat{r}=\delta_{n m} \frac{4 \pi}{2 n+1} P_{n}(\hat{x} \cdot \hat{y}) .
$$

As $\hat{y}$ is varied, the set of Legendre polynomials up to order $p-1$ span the set of all $\sum_{n=0}^{p-1} 2 n+1=p^{2}$ polynomials in $\hat{x}$ from degrees 0 through $p-1$ on the unit sphere. 28.

Because $K$ generates polynomials, functions on $S$ can be represented in terms of their values at a small set of quadrature points. We make use of standard quadrature formulas prescribing a set of $N \sim \frac{3}{2} p^{2}$ roots, $\hat{r}_{i}$, and associated weights, $w_{i}^{0}$, capable of integrating all polynomials up to order $2 p-2$. 228. Using such a quadrature set, for polynomial functions $\sigma$ of degree less than $p$,

$$
\sigma(\hat{x})=\int K(\hat{x}, \hat{y}) \sigma(\hat{y}) d y=\sum_{i=1}^{N} w_{i}^{0} \sigma\left(\hat{r}_{i}\right) K\left(\hat{x}, \hat{r}_{i}\right) .
$$

We will see that the coefficients $w_{i}^{0} \sigma\left(\hat{r}_{i}\right)$ act as the effective charges on the spherical surface. The numerical computations presented in Sections III and IV make use of the Lebedev quadrature set.29.

The choice of scale with $|x|$ and $|y|$ in Eq. 10 prescribes a default behavior off the surface of the unit sphere coinciding with that of Ref. 27.

$$
\begin{aligned}
& \int_{S} K(x, \hat{y}) O_{l m}(\hat{y}) d^{2} \hat{y}=O_{l m}(x) \\
& \int_{S} M_{l m}(\hat{x}) K(\hat{x}, y) d^{2} \hat{x}=M_{l m}(y) .
\end{aligned}
$$

These formulas can be used to directly translate formulas using spherical harmonics back into Legendre polynomials, as is done in Appendix A. Each choice of the coordinate axes for spherical harmonics of order $n$ generates $2 n+1$ harmonics, which correspond to a particular basis for the $2 n+1$ degenerate eigenfunctions of $P_{n}\left(r_{i} \cdot r_{j}\right)$. According to Eq. 11, each of the $2 n+1$ vectors has eigenvalue $4 \pi /(2 n+1)$. Working directly with $P_{n}$ avoids decomposition to this basis, removing angular variables from the resulting expressions.

The required separation of source and destination points ( $x$ and $y$ in Fig. 1) is easily achieved using the scaled reproducing kernel,

$$
\begin{aligned}
& \left(-y \cdot \partial_{x}\right)^{n}|x|^{-1} / n !=L_{n}(y, x) \\
& \quad=\int_{S} K(y / R, \hat{r}) L_{n}(R \hat{r}, x) d^{2} \hat{r}
\end{aligned}
$$

or

$$
=\int_{S} L_{n}(y, R \hat{r}) K(\hat{r}, x / R) d^{2} \hat{r} .
$$

Equation 15 generates the outer expansion by collecting the integration over sources inside the bounding sphere $(|y|<R)$ into an effective surface charge distribution,

$$
\sigma_{o}(R \hat{r}) \equiv \int \rho(y) K(y / R, \hat{r}) d^{3} y .
$$

The source's $n$-th order contribution to the potential at point $x$ is then

$$
\int L_{n}(y, x) \rho(y) d^{3} y=\int_{S} L_{n}(R \hat{r}, x) \sigma_{o}(R \hat{r}) d^{2} \hat{r} .
$$

Likewise, Eq. 16 generates the inner expansion when collecting the integration over sources outside the bounding sphere, $|x|>R$,

$$
\sigma_{i}(R \hat{r}) \equiv \int \rho(x) K(\hat{r}, x / R) d^{3} y .
$$

with the $n$-th order potential inside the sphere given by

$$
\int L_{n}(x, y) \rho(x) d^{3} x=\int_{S} L_{n}(x, R \hat{r}) \sigma_{i}(R \hat{r}) d^{2} \hat{r} .
$$




\section{B. Energy in the Quadrature Representation}

The interaction energy between two sets of point sources ( $y$ inside and $x$ outside of a sphere of radius $R$ ) is written in a multipole expansion as

$$
E=\sum_{x, y} \frac{q_{x} q_{y}}{|x-y|}=\frac{1}{R} \sum_{x, y, n=0} q_{x} q_{y} L_{n}(y / R, x / R)
$$

Using both the outer expansion projecting the enclosed charges onto a surrounding sphere $\left(L_{n}(y, x) \rightarrow\right.$ $\int_{S} K(y / R, \hat{r}) L_{n}(R \hat{r}, x) d^{2} \hat{r}$, Eq. 15, and the inner expansion projecting the outer charges onto the same sphere $\left(L_{n}(R \hat{r}, x) \rightarrow \int L_{n}(R \hat{r}, R \hat{s}) K(\hat{s}, x / R) d^{2} \hat{s}\right.$, Eq. 16,

$$
\begin{aligned}
E= & \frac{1}{R} \sum_{x, y, i, j, n=0} L_{n}\left(\hat{r}_{i}, \hat{r}_{j}\right) w_{i}^{0} w_{j}^{0} \\
& \times q_{y} K\left(y / R, \hat{r}_{i}\right) q_{x} K\left(\hat{r}_{j}, x / R\right) \\
= & \frac{1}{R} \sum_{i, j, n=0} w_{i}^{0} \sigma_{o}\left(R \hat{r}_{i}\right) w_{j}^{0} \sigma_{i}\left(R \hat{r}_{j}\right) L_{n}\left(\hat{r}_{i}, \hat{r}_{j}\right) .
\end{aligned}
$$

The second step uses the definitions in Eq. 17 and Eq 19 . In most cases, expressions involving the potential are simpler.

In addition, when $R$ is large, the energy is described equally well by treating the spherical quadrature points as point charge sources. This can be derived by inserting the moment shifting formula (Eq; 30 for $\sigma_{i}\left(R \hat{r}_{j}\right) \rightarrow$ $\sum_{k} K\left(\hat{r}_{j}, \frac{R_{x} \hat{r}_{k}-t}{R}\right) w_{k}^{0} \sigma_{o}\left(R \hat{r}_{k} ; t\right)$ and summing over $j$ to show

$$
\begin{aligned}
E & =\sum_{i, k, n} w_{i}^{0} \sigma_{o}\left(R \hat{r}_{i}\right) w_{k}^{0} \sigma_{o}\left(R_{x} \hat{r}_{k} ; t\right) L_{n}\left(R \hat{r}_{i}, R_{x} \hat{r}_{k}-t\right) \\
& \simeq \sum_{i, k} \frac{w_{i}^{0} \sigma_{o}\left(R \hat{r}_{i}\right) w_{k}^{0} \sigma_{o}\left(R_{x} \hat{r}_{k} ; t\right)}{\left|R_{x} r_{k}-t-R \hat{r}_{i}\right|}
\end{aligned}
$$

\section{Outline}

Much of the work focused on real-space (Cartesian) representations of the expansion (Eq. 9p uses a tensor notation. The set of three-dimensional tensors of orders $\mathrm{n}, \sum_{i} q_{i} r_{i}^{(n)}$, from $n=0$ up to order $n=p-1$ is termed a 'Cartesian polytensor.' Appendix B shows a direct correspondence between Cartesian polytensors and polynomial functions with maximum degree $p-1$. It is shown that outer and inner products between supersymmetric tensors have much simpler statements in terms of polynomial multiplication and differentiation. Moreover, the set of trace-free Cartesian polytensors [11, 14 is exactly identified with the set of polynomials on the unit sphere, $\sigma(\hat{x})$. In combination with the representation theorem, Eq. 12, these identities completely connect tensor and harmonic representations to a novel, quadrature representation of polytensor space.
Section III illustrates the simplicity of the quadrature method by testing moment shift formulas used during the fast multipole method. Surprisingly, these have the same form as the initial moment fitting. Moreover, it shows that the weights for the quadrature representations exactly coincide with point charges that reproduce those multipole moments at sufficient distance from the bounding sphere. As expected by the exact correspondence to spherical harmonics, numerical results show that the error of representing random point sources scales with distance identically to traditional spherical harmonic multipoles. Section IV provides exact quadratures for the single and double-layer potentials on a spherical surface. Numerical results in Sec. IV] demonstrate a singularity representation for multiple spheres interacting through a perfect, irrotational fluid.

The conclusion summarizes the connections created here and outlines new applications and simplifications that can be tackled in future work.

\section{CONVERSION BETWEEN QUADRATURE AND CARTESIAN TENSORS}

Although the quadrature representation is a complete, self-contained basis for expressing multipole moments and externally imposed fields, comparison with existing literature requires translation between Cartesian representations.

Writing the order- $n$ Cartesian multipole moment tensor as $M^{(n)} \equiv \int y^{(n)} \rho(y) d^{3} y$, the directional moment is defined as the complete ( $n$-way) tensor contraction between $M^{(n)}$ and a test vector, $r$,

$$
r_{(n)}^{(n)} M^{(n)}=\int(r \cdot y)^{n} \rho(y) d^{3} y .
$$

Complete information about the polytensor, $\left\{M^{(n)}, n=0, \ldots, p-1\right\}$ is contained in the set of directional moments against the quadrature points, $\left\{\hat{r}_{i}\right\}$ (see Appendix B for details). These directional moments can be substituted wherever powers of $(\hat{r} \cdot y)$ appear in the moment matching equation 17 .

Conversion back to Cartesian form is also simple, since the quadrature weights were defined to reproduce polynomial integrals, which include all (trace-free) tensors,

$$
\int \mathcal{D}_{n} r^{(n)} \rho(r) d^{3} r=\sum_{i} w_{i}^{0} \sigma\left(\hat{r}_{i}\right) r_{i}^{(n)}, n<p .
$$

Here, $\mathcal{D}_{n}$ is the de-tracer projection of Ref. 14 defined by

$$
\hat{x}^{(n)}{ }_{(n)} \mathcal{D}_{n} \hat{y}^{(n)}=\frac{n !}{(2 n-1) ! !} P_{n}(\hat{x} \cdot \hat{y})
$$

These formulas show that the conversion to quadrature form is one way to project a polynomial into a trace-free form. Alternatively, trace-free Cartesian tensors simplify 
Eq. 17, since Eq. 26 can be used in $K$, (Eq. 10)

$$
\begin{aligned}
\sigma_{o}(R \hat{r})=\sum_{n} & \frac{(2 n+1)}{4 \pi} \frac{(2 n-1) ! !}{n !} \\
& \times \hat{r}^{(n)}(n) \mathcal{D}_{n} M^{(n)} R^{-n} .
\end{aligned}
$$

This sum includes only the leading terms of $L_{n}$ at each order, $n$.

\section{FMM OPERATIONS}

In this section, we elaborate the translation formulas for the quadrature representation of multipoles. We achieve greater generality in comparison with previous Cartesian formulations [30, by working directly with the moment space, rather than the potential. In comparison with black-box fast multipole methods, 31 the availability of spherical quadrature rules justifies the use of Legendre polynomials because they represent moment space - regardless of their specific role in the Poisson problem. For a detailed introduction to the fast multipole method, see Ref. [32.

The outer expansion is defined by the moments of the enclosed charge distribution. The moments of the shifted distribution should coincide with those of the original. This can be shown from

$$
\sigma_{o}\left(R_{1} \hat{r} ; x_{1}\right)=\int_{S} K\left(\frac{R_{0} \hat{s}+x_{0}-x_{1}}{R_{1}}, \hat{r}\right) \sigma_{o}\left(R_{0} \hat{s} ; x_{0}\right) d^{2} \hat{s} .
$$

The notation here is that $\sigma_{o}\left(R_{1} \hat{r} ; x_{1}\right)$ represents the outer expansion about the origin $x_{1}$, defined on the set of points at a distance $R_{1}$ from $x_{1}$. Since both outer expansions are polynomials of maximum degree $p-1$ in $R \hat{r}$, the reproducing kernel, $K$ is able to duplicate them from any appropriate set of quadrature points.

The inner expansion is defined by the derivatives of the potential about the origin. This makes translation troublesome because the expansion about a new point does not necessarily need to obey the trace-free condition. An implicit argument can be used to show that propagating the inner expansion to the potential on a small sphere around the new expansion point can be used to find charges on a new enclosing sphere that duplicate this potential up to the same order as the original expansion. The net result for the inner expansion is the same as the initial fitting,

$$
\sigma_{i}\left(R_{1} \hat{r} ; x_{0}+t\right)=\int K\left(\hat{r}, \frac{R_{0} \hat{s}-t}{R_{1}}\right) \sigma_{i}\left(R_{0} \hat{s} ; x_{0}\right) d^{2} \hat{s}
$$

or

$$
=\int K\left(\hat{r}, \frac{R_{0} \hat{s}-t}{R_{1}}\right) \sigma_{o}\left(R_{0} \hat{s} ; x_{0}\right) d^{2} \hat{s} .
$$

Both outer $\rightarrow$ inner and inner $\rightarrow$ inner shift operators give the same equation. In fact, all shift operators arriving at an outer expansion are identical to the initial
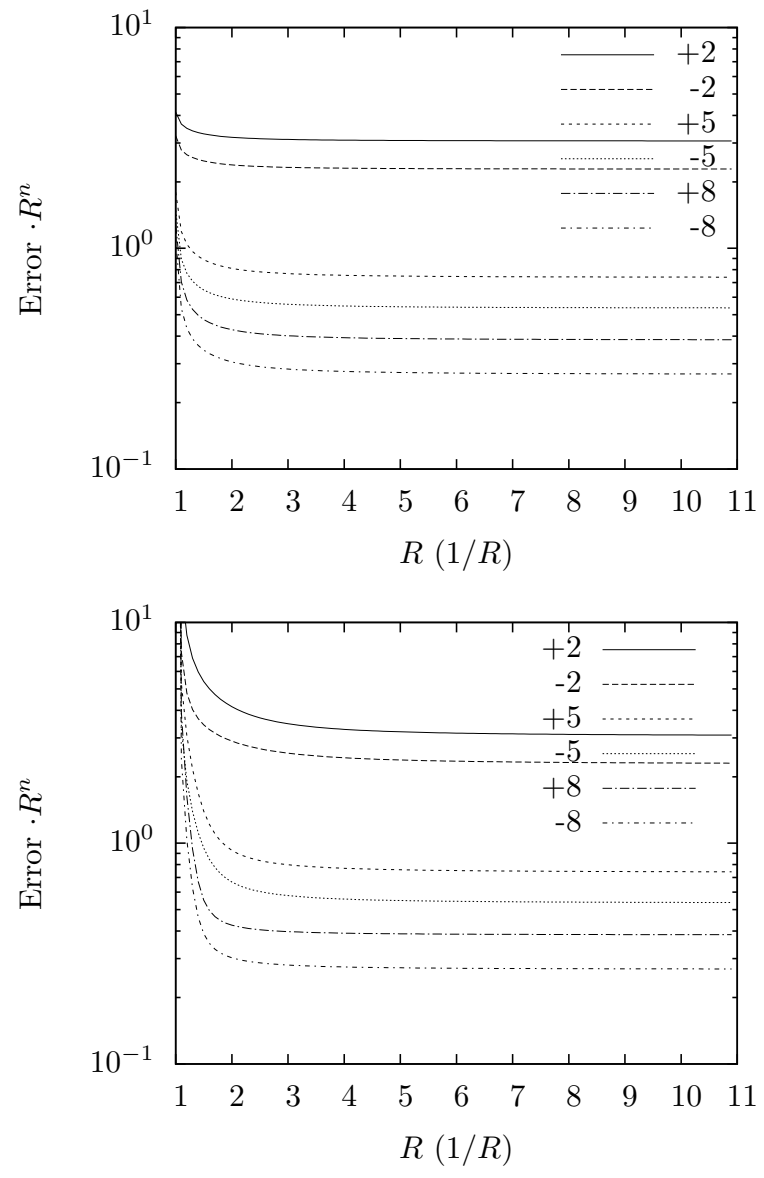

FIG. 2. Pre-factors for the multipole representation error. The residual is scaled by $r^{p+1}$ (outer expansion, labeled $+p$ ) or $r^{-p}$ (inner expansion, labeled $-p$ ). The left panel shows the error of exact quadrature using Eq. 32 or Eq. 34. while the right shows the error of summing $1 / r$, treating the quadrature points as point sources. Point charge sources make excellent representations of multipole sources and external fields.

moment matching (Eq. 17). All shift operators arriving at an inner expansion are also identical to the inverse moment matching (Eq. 19). Appendix A shows that these formulas are identical to traditional expressions involving spherical harmonics.

The only caveat that appears here is that the kernel, $K(\hat{r}, \hat{s}-t)$, contains singularities when $|t|=1$. This problem enters because the shifting formula is only welldefined when $t$ does not approach the sphere bounding the source charge distribution. This motivates the introduction of the scaled distributions in Eq. 17. The use of unscaled moments in shift formulas based on sphericalharmonics, while convergent for finite sums, might therefore be expected to show numerical issues in the limit of large expansion order, $p$. 30, 33.

Figure 2 shows that the numerical error of the quadrature representation, including appropriate bounding radii, have the same power-law decrease in error as previously shown for spherical harmonics. It plots the 
accuracy of representing the electrostatic potential as a function of distance from the bounding sphere. A set of 4000 point charges assigned from a uniform distribution between -1 and +1 were placed uniformily in the cube $\left[-\frac{\sqrt{3}}{3}, \frac{\sqrt{3}}{3}\right]^{(3)}$. The residual error per point shown was averaged over 100 charge distributions and over the 86 points of the Lebedev quadrature grid of order 15 on the surface of the evaluation sphere at varying distance, $r$, from the bounding sphere. The inner expansion has an inverted geometry, with the sources scaled by $1 /|x|^{2}$ to put them outside the sphere, and the evaluation test points likewise inverted to the inside, shrinking toward $r=0$.

For comparison, the simple summation of $\sum_{i} w_{i} /(r-$ $R \hat{r}_{i}$ ) using the quadrature weights as charges is shown in the right panel of Fig. 2. The pre-factor for both error curves converges at a radius above 3 , showing that the quadrature-based representation gives highly accurate point charges that simultaneously represent all multipoles. Practically, this solves the problem of placing $O\left(p^{2}\right)$ discrete charges to mimic all multipolar moments up to arbitrary order posed in Refs. [1, 13, 18]. This is also the reason for the symmetry of the shifting formulas - the weights are arrived at through the same process of fitting the inner or outer expansion.

Figure 3 shows the error in multipole shifting operations, plotted as a function of angle from the shift direction. For the outer expansion, the test points are fixed at $R=2$, while the source distribution is shifted to the right by $0.2,0.6$, and 0.8 . The locations of the source points were scaled down for each shift to maintain contact of the rightmost face of the source cube with the unit bounding sphere. Summation of $\sum_{i} w_{i} /\left(r-\hat{r}_{i}\right)$ from the representation weights had nearly identical error (not shown). For the inner expansion, the source points were inverted to lie outside the sphere and remain fixed. The evaluation sphere started at the origin with $R=1 / 2$, and was successively shifted in the $x$-direction by $0.1,0.2,0.3$, and 0.4 , with the radius scaled down to maintain contact between the rightmost point of original evaluation sphere and the shifted version.

The error of the inner expansion shows much more variation as a function of the cosine with respect to the shift direction, since the distance to the unit sphere is more quickly varying than in the outer expansion geometry. The scaling of the outer expansion with expansion order, $p$, also appears somewhat better because the source locations were scaled down with increasingly large shifts in that geometry.

\section{SINGULARITY METHODS}

The boundary integral method utilizes the fact that in regions, $\Omega$, where $\mathcal{L} \phi=0$, the potential can be expressed as a boundary integral using the divergence theorem,

$$
\begin{aligned}
\phi(x)=\int_{\partial \Omega} \phi(y) & \cdot p(y) \partial_{y} G(x-y) \\
& -G(x-y) n \cdot p(y) \partial_{y} \phi(y) d^{2} y .
\end{aligned}
$$

Here $n$ is the inward-pointing normal vector on the surface at point $y$, and a Sturm-Liouville form has been assumed for $\mathcal{L} G \equiv \partial \cdot(p \partial G)-u G=\delta(r)$. The normal vector directions are shown in Fig. 1. This equality makes it possible to solve $\mathcal{L} \phi=0$ with constant potential or constant flux boundary conditions. 19]

Boundary integrals over polynomial distributions on the sphere, $\sigma$, can be computed exactly by the summations,

$$
\begin{aligned}
\int_{S}|R \hat{r}-x|^{-1} \sigma(R \hat{r}) d^{2} \hat{r} & =\sum_{m=0, i}^{p-1} L_{m}\left(R \hat{r}_{i}, x\right) w_{i}^{\sigma} \\
\int_{S}\left[\hat{r} \cdot \partial_{r}|R \hat{r}-x|^{-1}\right] \sigma(R \hat{r}) d^{2} \hat{r} & =\sum_{m=0, i}^{p-1} \frac{m}{R} L_{m}\left(R \hat{r}_{i}, x\right) w_{i}^{\sigma} \\
\int_{S}|R \hat{r}-y|^{-1} \sigma(R \hat{r}) d^{2} \hat{r} & =\sum_{m=0, i}^{p-1} L_{m}\left(y, R \hat{r}_{i}\right) w_{i}^{\sigma} \\
\int_{S}\left[\hat{r} \cdot \partial_{r}|R \hat{r}-y|^{-1}\right] \sigma(\hat{r}) d^{2} \hat{r} & =-\sum_{m=0, i}^{p-1} \frac{m+1}{R} L_{m}\left(y, R \hat{r}_{i}\right) w_{i}^{\sigma}
\end{aligned}
$$

For $|y|<R$ and $|x|>R$, and where $w_{i}^{\sigma} \equiv w_{i}^{0} \sigma\left(R \hat{r}_{i}\right)$. These can be proven by expanding $|r-x|^{-1}$ in orthogonal polynomial spaces, $L_{n}$, to give an integral over $S$ and then writing that integral as a quadrature, exact for polynomial $\sigma$ of degree less than $p$. Equations 32 and 34 are just the inner and outer expansions.

Equations 33 and 35 give the complete, singular surface integral, not just the Cauchy principal value part. 19. 

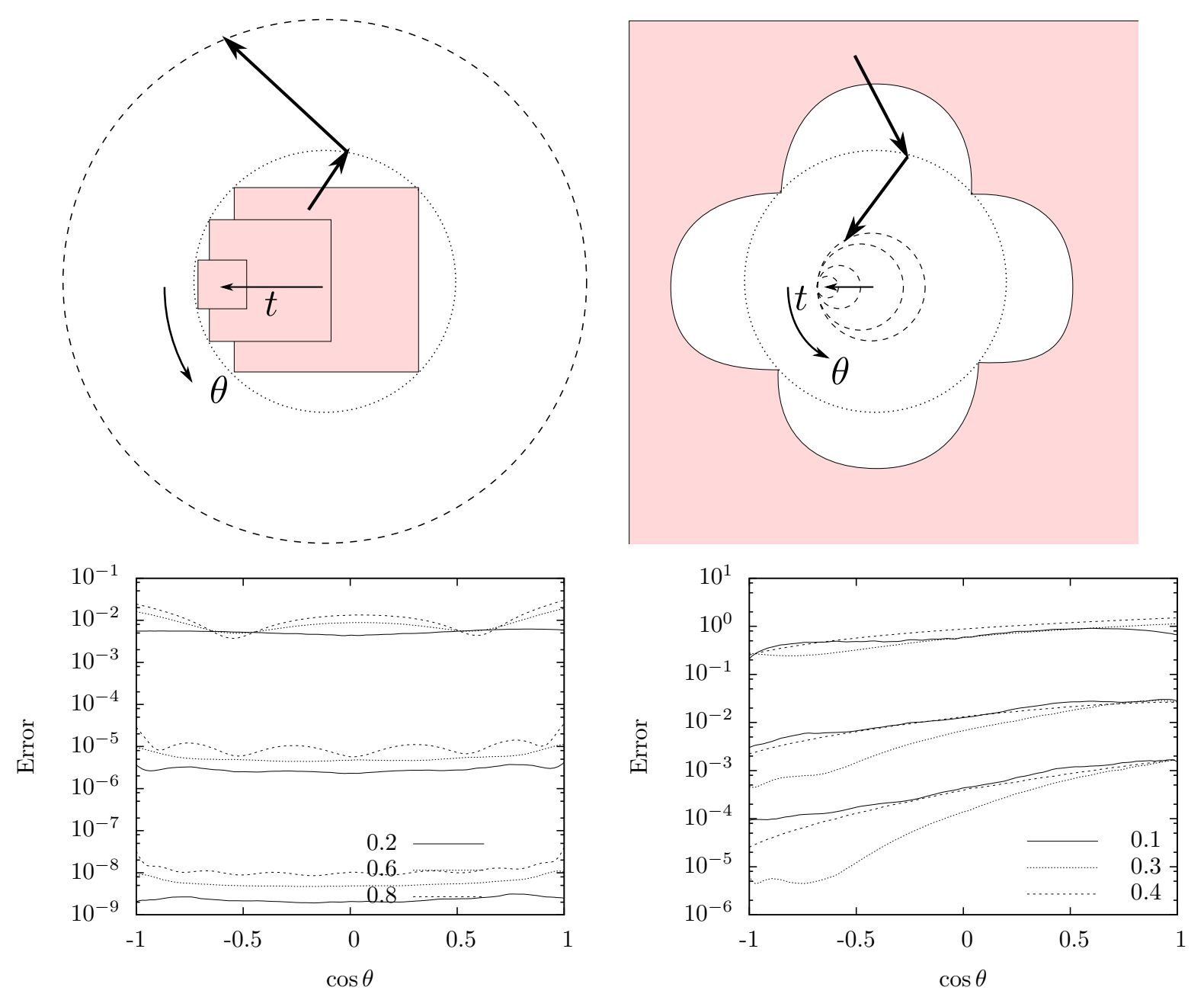

FIG. 3. Absolute multipole representation error in the potential for shifted source distributions in the outer expansion $(|x|=2$, left panel) or shifted evaluation spheres in the inner expansion $(|y|=1 / 2$, right panel). Graphics above each indicate the source locations, distributed randomly throughout the shaded areas, and evaluation points using dashed lines. The dotted circle indicates the bounding sphere at $R=1$. Both are plotted as a function of $\cos \theta=\hat{r} \cdot \hat{t}$, where $\hat{t}$ is the shift direction. The magnitude of the shifts are shown in the figure legend. Expansion orders are 2, 5, and 8 as in Fig. 2 Increasing expansion orders show up as groups of curves with decreasing error.

This can be seen by noting that the jump discontinuity in $\hat{r} \cdot \partial \phi$ as both $x$ and $y$ approach the surface point, $y$ is

$$
\begin{aligned}
& \left.F_{\text {ext }}\right|_{x=y}-F_{\mathrm{int}} \\
& =R^{2} \int_{S} \hat{r} \cdot \partial_{r} \frac{1}{|R \hat{r}-x|} \sigma(R \hat{r})-\hat{r} \cdot \partial_{r} \frac{1}{|\hat{r}-y|} \sigma(R \hat{r}) d^{2} \hat{r} \\
& =4 \pi R \sum_{n} \frac{2 n+1}{4 \pi} L_{n}\left(R \hat{y}, R \hat{r}_{i}\right) w_{i}^{0} \sigma\left(R \hat{r}_{i}\right)=4 \pi \sigma(y) .
\end{aligned}
$$

The last equality comes from recognizing the expression for the reproducing kernel (Eq. 10), which scales as $R^{n}$ in its first argument and $R^{-n-1}$ in its second.

For a set of spheres moving through an incompressible, irrotational fluid, the flow at every point in the fluid can be written as the derivative of a potential,

$$
\begin{aligned}
v(x) & \equiv-\partial \Phi(x) \\
\partial^{2} \Phi(x) & =0 .
\end{aligned}
$$

Each point on a spherical boundary gives a constant field condition,

$$
n \cdot v_{0}=-n \cdot \partial \Phi(x) .
$$

The solution can be found numerically using a pointbased, quadrature representation of $\Phi$, so that Eq. 31 becomes the linear equation, $(I-F) \cdot \Phi=G \cdot\left(n \cdot v_{0}\right)$.

Figure $4 \mathrm{~s}$ top panel shows the motion of three spheres through an incompressible 3D fluid. Point singularities represented using quadrature multipole expansions on the surfaces of the spheres show exponential convergence. The smaller spheres have radius 1 , are located at $x=-1$ 

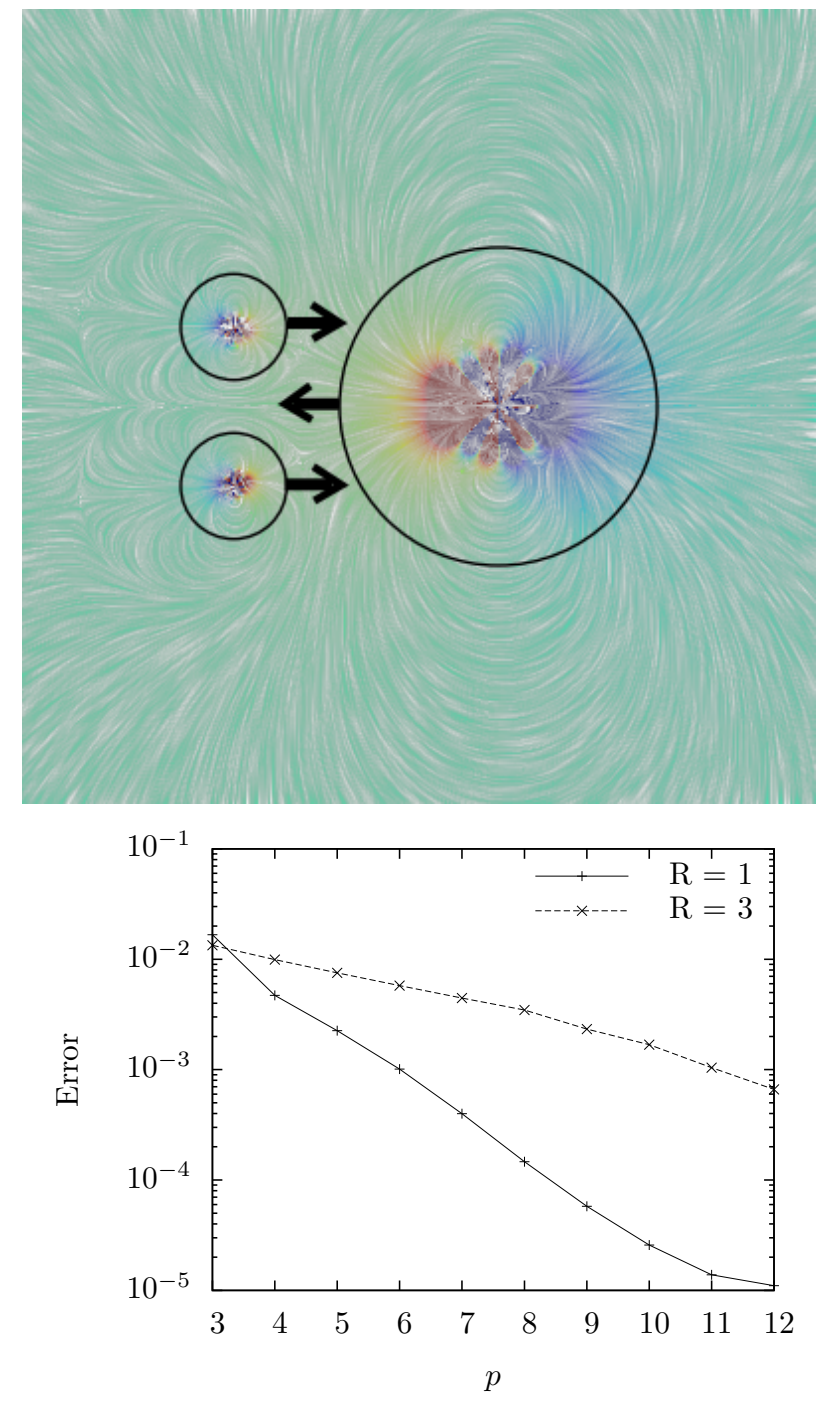

FIG. 4. Multipole representation of potential flow for three spheres in the $z=0$ plane (upper panel, $p=8$ ). Color indicates the potential field. The flow velocity was rendered using line integral convolution. The lower panel shows the surface-averaged boundary error divided into contributions from the larger and smaller spheres.

and $y= \pm 3 / 2$ and are traveling with unit velocity in the $+x$ direction. The larger sphere has radius 3 , is located on the $y$ axis at $x=4$, and is traveling with unit velocity in the $-x$ direction. The velocity at every point in the fluid is calculated using Eq. 38 from a sum of the three multipoles (outer expansion of Eqns. 32 and 33). Weights on the quadrature set over each sphere were solved to fix the boundary condition, Eq. 39 at each point in the quadrature set.

Error in the normal derivative boundary condition (lower panel of Figure 4 was evaluated at the spherical surfaces using a larger Lebedev quadrature grid of order 59 with 1202 points. As the quadrature order $(p)$ is increased, more points are added to the multipolar expansion on each sphere, and the discrepancy between the computed and imposed normal velocity decreases exponentially.

\section{DISCUSSION}

An isomorphism between symmetric Cartesian tensors and polynomial functions is used to show an optimal representation for polytensors in terms of real weights, $w_{i}$, on a fixed set of basis vectors, $\left\{r_{i}\right\}$. This equivalence is compactly expressed in terms of equivalent representations of the moment integrals,

$$
\begin{aligned}
r_{(n)}^{(n)} \int x^{(n)} \rho(x) d^{3} x & =\int(r \cdot x)^{n} \rho(x) d^{3} x \\
& =\sum_{i}^{N}\left(r \cdot r_{i}\right)^{n} w_{i} .
\end{aligned}
$$

Three numerical results were presented. First, we showed the numerical error in matching spherical moments of a cloud of discrete point charges. We compared the exact Eq. 32 with the numerical summation over the scaled quadrature points, $R \hat{r}_{i}$. These made use of Lebedev quadrature rules 29] tabulated up to order 131 by Burkardt. 34] Next, we showed that the expressions for translating the origin of inner and outer moment expansions have the same error as the corresponding translation formulas using spherical harmonics. Appendix A gives further details on the mathematical translation. Finally, we presented convergence results for FMM solutions to potential flow between multiple interacting spheres. The central role of the quadrature representation in all these methods allows us to skip over consideration of an intermediate spherical harmonic representation.

When used in a Taylor expansion of a scale-invariant Green's function, $G(c r)=c^{\alpha} G(r)$, the $r$-dependence of the solutions are fixed, and only a subset of the full moment space is required. That subspace is equivalent to the set of polynomials on the unit sphere. This condition reduces the $\left(\begin{array}{c}n+2 \\ n\end{array}\right)$ polynomials at each total degree $n$ to only $2 n+1$. Approximation of integrals involving $G$ up to order $p-1$ thus requires only $N=p^{2}$ coefficients. Convenient expressions for finding these coefficients from a source distribution, translation operators, and integrals involving expansions of the Poisson kernel, $|r|^{-1}$ were given. Numerical results verified that the outer expansions have error $r^{-p-1}$, and the inner expansions have error $r^{p}$, consistent with the corresponding spherical harmonic formulas.

The computational scaling of naïvely evaluating the moment translation formula Eq. 28 is $p^{4}$. This cost scales as $p^{3}$ for spherical harmonic translations that perform rotations to align the translation axis with the azimuthal reference axis, $\hat{z}$. 35. Similar savings can be realized using the quadrature-based scheme, when using equally spaced points along rings spaced vertically according to Gauss-Legendre 36] or Gauss-Jacobi 37] 
quadrature. Fast, $O\left(p^{2} \log p\right)$, transformations between harmonics and the Gauss-Jacobi quadrature representation are available. 37, 38. Further, rotations that make use of these transformations can achieve $O\left(p^{3}\right)$ scaling or, using approximate algorithms for matrix sparsification, $O\left(p^{2} \log p\right)$. 36] Since the matrix in Eq. 28, $\left[K\left(\frac{R_{0} \hat{r}_{j}+t}{R_{1}}, \hat{r}_{i}\right)\right]_{i j}$ has as many unique elements as unique cosines, $\hat{r}_{i} \cdot \hat{r}_{j}$, quadrature rules like those above containing $O(p)$ vertical rings have a translation scaling as $O\left(p^{2}\right)$ after rotation.

The Cartesian representations found in this work generalize and extend analogous results found using manually tabulated spherical harmonics. The decomposition of Legendre polynomials into spherical harmonics (Eq. 5 shows that the harmonics correspond to particular choices for the eigenfunctions of $P_{n}$. To work with this set, the matrices $\left[P_{n}\left(\hat{r}_{i}, \hat{r}_{j}\right) \sqrt{w_{i}^{0} w_{j}^{0}}\right]$ can be numerically diagonalized. This gives rise to $2 n+1$ eigenvectors for each $P_{n}$, with the same eigenvalue, $4 \pi /(2 n+1)$. Using the path from tensors to quadrature points to harmonics, results found using Cartesian polytensors can be translated to spherical harmonics and vice-versa. Moreover, Cartesian tensor contractions which were naïvely $O\left(3^{p}\right)$ have been reduced to an efficient summation over an $O\left(p^{2}\right)$ point set.

For differential equations not respecting scaleinvariance, the representation of $\rho$ via the polynomial form of its moments still provides a simple path for deriving accurate numerical methods. Using any $\sum_{n}\left(\begin{array}{c}n+2 \\ n\end{array}\right)$ points for which the space of directional moments, $\left[c_{K}\left(r_{i} ; r_{j}\right)\right]$, has full rank, a reproducing kernel and polynomial interpolation formulas can be found through numerical inversion of $\left[c_{K}\left(r_{i} ; r_{j}\right)\right]$. [39] Of course, considering only the moments actually appearing in the expansion of $G$, using an optimal quadrature rule, and accelerating the evaluation of the moments by exploiting symmetry will reduce the computational overhead of this exercise.

Real-space expressions are the most desirable starting point for deriving fast algorithms. First, all parts of the computation have a regular, vector structure, making simplifying optimizations for parallel hardware. Second, high-level form of the matrix operations and lack of angular coordinates makes derivations much simpler. Not only are the full set of (already well-developed and efficient) spectral methods available for fast implementation, but also new symmetry-based, sparse factorizations remain to be explored. 40. Sparse factorization is at the heart of fast Fourier transformation methods. Finally, stable, $O\left(p^{3}\right)$ rotation formulas using point-based evaluation of the reproducing kernel (Eq. 10 ) have been shown and critically tested in Ref. [33]. Even still, a large fraction of computation time in that work was spent on evaluating spherical harmonic functions on the set of rotated real-space points. Either of these optimizations would reduce the overall scaling to $O\left(p^{3}\right)$, recovering the scaling of rotation-based translations for spherical harmonics. 41.

\section{CONCLUSIONS}

The representations found in this work greatly simplify the analysis and numerical use of distribution functions on a sphere. Many of the optimizations for spherical harmonics rely on the symmetry of the spherical harmonic functions. Future work should consider alternate quadrature sets that directly exploit real-space symmetry to reduce the scaling of these methods with expansion order. Working with distribution functions on the sphere also presents a new approach to tensor analysis, traditional boundary value problems and extensions of black-box multipole methods to potentials governed by more complicated PDEs.

Three novel results that come from this connection are space-optimal, vector-based representations for Cartesian polytensors, an exact representation of point multipoles using discrete point charges, and simplification of the moment shifting formulas for FMM.

\section{ACKNOWLEDGMENTS}

Support for this work was provided by the USF Foundation. Line integral convolution routines used here were developed by Anne Archibald based on the work of Cabral, Brian and Laeith Leedom (SIGGRAPH '93: 263$270,1993)$, and wrapped in scipy 42 by David Huard.

\section{Appendix A: Comparison to Spherical Harmonic Expressions}

The expressions given in Sec. III are real-space counterparts of the outer and inner expansions obtained in the spherical harmonic formalism (Eq. 2), 27]

$$
\begin{aligned}
\omega_{l m}(Q ; A) & \equiv \int \rho(y) O_{l m}(y) d^{3} y \\
& =\int_{S} O_{l m}(\hat{r}) \sigma_{o}(\hat{r}) d^{2} r \\
\sigma_{o}(\hat{r}) & =\sum_{l=0}^{p-1} \sum_{m=-l}^{l} \frac{2 l+1}{4 \pi} M_{l m}(\hat{r}) \omega_{l m}(Q ; A) \\
\mu_{l m}(Q ; A) & \equiv \int_{m} \rho(y) M_{l m}(y) d^{3} y \\
& =\int_{S} M_{l m}(\hat{r}) \sigma_{i}(\hat{r}) d^{2} r \\
\sigma_{i}(\hat{r}) & =\sum_{l=0} \sum_{m=-l}^{l} \frac{2 l+1}{4 \pi} O_{l m}(\hat{r}) \mu_{l m}(Q ; A) .
\end{aligned}
$$

As explained in Sec. I the integrals on $S$ can be carried out with exact summations over any $O\left(p^{2}\right)$ points using quadrature methods capable of integrating all polynomials on $S$ up to order $2 p-2$. Both the number of degrees of freedom and the set of moments that can be 
represented match between quadrature and spherical harmonic methods. In addition, the naïve implementation of both methods has the same scaling. However, where harmonics require computation of $p^{2}$ basis functions, $O_{n m}$, the quadrature method only requires computation of the $p$ Legendre polynomials, $L_{n}$.

The shift formula of Eq. 28 coincides with Ref. 27, as can be seen from the translations in Eq. A1

$$
\begin{aligned}
\sigma_{o}\left(R_{1} \hat{r} ; x_{1}\right) & =\sum_{l m} \frac{2 l+1}{4 \pi} M_{l m}(\hat{r}) \sum_{j k}^{l} A_{j k}^{l m}\left(\left(x_{0}-x_{1}\right) / R_{1}\right) \int_{S} O_{j k}\left(\frac{R_{0}}{R_{1}} \hat{s}\right) \sigma_{o}\left(R_{0} \hat{s} ; x_{0}\right) d^{2} \hat{s} \\
& =\int_{S} K\left(\frac{R_{0} \hat{s}+x_{0}-x_{1}}{R_{1}}, \hat{r}\right) \sigma_{o}\left(R_{0} \hat{s} ; x_{0}\right) d^{2} \hat{s}
\end{aligned}
$$

since $A_{j k}^{l m}$ was defined so that

$$
\sum_{j k}^{l} A_{j k}^{l m}(t) O_{j k}(a)=O_{l m}(a+t)
$$

To derive the other shift operators, define $B$ and $C$ as

in Ref. [27] so that

$$
\begin{aligned}
& M_{l m}(a-t)=\sum_{j k}^{\infty} B_{j k}^{l m}(t) O_{j k}(a) \\
& M_{l m}(a-t)=\sum_{j k}^{\infty} C_{j k}^{l m}(t) M_{j k}(a),
\end{aligned}
$$

and note that

$$
\begin{aligned}
\sigma_{i}\left(R_{1} \hat{r} ; x_{0}+t\right) & =\sum_{l m} \frac{2 l+1}{4 \pi} O_{l m}(\hat{r}) \int \sum_{j k}^{\infty} B_{j k}^{l m}\left(t / R_{1}\right) O_{j k}\left(\frac{R_{0}}{R_{1}} \hat{s}\right) \sigma_{o}\left(R_{0} \hat{s} ; x_{0}\right) d^{2} \hat{s} \\
& =\int K\left(\hat{r}, \frac{R_{0} \hat{s}-t}{R_{1}}\right) \sigma_{o}\left(R_{0} \hat{s} ; x_{0}\right) d^{2} \hat{s} \\
\sigma_{i}\left(R_{1} \hat{r} ; x_{0}+t\right) & =\sum_{l m} \frac{2 l+1}{4 \pi} O_{l m}(\hat{r}) \int \sum_{j k}^{\infty} C_{j k}^{l m}\left(t / R_{1}\right) M_{j k}\left(\frac{R_{0}}{R_{1}} \hat{s}\right) \sigma_{i}\left(R_{0} \hat{s} ; x_{0}\right) d^{2} \hat{s} \\
& =\int K\left(\hat{r}, \frac{R_{0} \hat{s}-t}{R_{1}}\right) \sigma_{i}\left(R_{0} \hat{s} ; x_{0}\right) d^{2} \hat{s} .
\end{aligned}
$$

\section{Appendix B: Cartesian Tensors}

This section derives several relations showing the identity between supersymmetric tensors of order $n$ and homogeneous polynomials of the same order. These prove that relations involving such tensors can be stated identically in terms of polynomial functions. Tensors are tra- ditionally introduced via the Taylor expansion,

$$
\begin{aligned}
G(r) & =\left.\sum_{n=0}^{\infty} \frac{1}{n !}\left(r-r_{0}\right)^{(n)}(n) \partial_{r}^{(n)} G(r)\right|_{r=r_{0}} \\
& =\left.\sum_{n=0}^{\infty} \frac{1}{n !}\left(\left(r-r_{0}\right) \cdot \partial_{s}\right)^{n} G(s)\right|_{s=r_{0}},
\end{aligned}
$$

where variables with parenthesized superscripts denote tensors. Vectors are always written in lower-case. The $n$ - 
fold tensor contraction between $A^{(a)}$ and $B^{(b)}$ is denoted as $A^{(a)}(n) B^{(b)}$.

\section{Translations between polynomials and symmetric tensors}

We define the $n^{\text {th }}$ order directional moment of a threedimensional distribution, $\rho$, centered at the origin as the quantity,

$$
m_{n}(r)=\int(r \cdot x)^{n} \rho(x)\left|d^{3} x\right|
$$

The distribution need not be positive, so $\rho\left|d^{3} x\right|$ is a signed measure. It is clear from this definition that $m_{n}(r)$ is a homogeneous polynomial of order $n$, with the monomial expansion,

$$
\begin{array}{r}
m_{n}(r)=\sum_{n_{1}+n_{2}+n_{3}=n}\left(\begin{array}{c}
n \\
n_{1} n_{2} n_{3}
\end{array}\right) r_{x}^{n_{1}} r_{y}^{n_{2}} r_{z}^{n_{3}} \\
\times M\left[n_{1}, n_{2}, n_{3}\right],
\end{array}
$$

where $M\left[n_{1}, n_{2}, n_{3}\right]$ are the monomial moments of $\rho$. Since the test monomials, $x^{n_{1}} y^{n_{2}} z^{n_{3}}$ form a linearly independent set, $m_{n}(r)$ spans the complete set of $\left(\begin{array}{c}n+2 \\ n\end{array}\right)$ homogeneous polynomials in $r$. These moments are identical to Applequist's reduced notation for the supersymmetric tensor, $M^{(n)}=\partial_{r}^{(n)} m_{n}(r) / n !=\int x^{(n)} \rho(x)\left|d^{3} x\right|$.14 Alternatively, to every order $n$ supersymmetric tensor, $A^{(n)}$, there corresponds an order $n$ homogeneous polynomial,

$$
a_{n}(r)=r^{(n)}(n) A^{(n)} .
$$

The symmetric tensor (outer) product can be found very easily using these polynomials as,

$$
r^{(n+m)}{ }_{(n+m)} \mathcal{S}\left[A^{(n)} \otimes B^{(m)}\right]=a_{n}(r) b_{m}(r),
$$

where the symmetrizing operator, $\mathcal{S}$, averages over all $(n+m)$ ! permutations of the $n+m$ tensor indices. This formula can be proven by noting that

$$
\begin{aligned}
r_{(n+m)}^{(n+m)} & \left(A^{(n)} \otimes B^{(m)}\right) \\
& =\left(r^{(n)}(n) A^{(n)}\right)\left(r^{(m)}(m) B^{(m)}\right)
\end{aligned}
$$

for any permutation of the indices, since $r^{(n+m)}$ is symmetric. The tensor contraction (inner product) is, 39, 43.

$$
A^{(n)}{ }_{(n)} B^{(n)}=a_{n}\left(\partial_{r}\right) b_{n}(r) / n !
$$

These form a complete set of operations, since supersymmetric tensors can be built from multiplying polynomials (forming symmetric tensor products), and partial contractions can be found via differentiation, as

$$
\begin{aligned}
r_{(m)}^{(m)} & \left(A_{(n)}^{(n)} B^{(n+m)}\right) \\
& =\left(A^{(n)} \otimes r^{(m)}\right){ }_{(n+m)} B^{(n+m)} \\
& =\frac{1}{(n+m) !}\left(r \cdot \partial_{s}\right)^{m} a_{n}\left(\partial_{s}\right) b_{n+m}(s) \\
& =\frac{1}{(n+m) !} r^{(m)}{ }_{(m)} \partial_{s}^{(m)} a_{n}\left(\partial_{s}\right) b_{n+m}(s) \\
& =\frac{m !}{(n+m) !} a_{n}\left(\partial_{r}\right) b_{n+m}(r) .
\end{aligned}
$$

These identities are even simpler to manipulate when expressed in terms of moment integrals,

$$
\begin{aligned}
& r_{(m)}^{(m)}\left(A_{(n)}^{(n)} B^{(n+m)}\right) \\
& =\iint(x \cdot y)^{n}(r \cdot y)^{m} \rho_{A}(x) \rho_{B}(y)\left|d^{3} x \| d^{3} y\right| \\
& =\frac{m !}{(n+m) !} \iint\left(x \cdot \partial_{r}\right)^{n}(r \cdot y)^{n+m} \rho_{A}(x) \rho_{B}(y)\left|d^{3} x \| d^{3} y\right| .
\end{aligned}
$$

This expansion always valid, since a signed measure yielding any tensor $M\left[n_{1}, n_{2}, n_{3}\right]$ (by Eq. B2 always exists due to the linear independence of the monomials mentioned above.

Supersymmetric cartesian tensors and polynomial functions where each term has the same degree (termed projective polynomials), are thus in 1:1 correspondence. A suitable quadrature formula for polynomials could reduce integrals such as the above to summation over point sets.

In this work, we restrict our attention to polynomial functions defined over the surface of a sphere. Because the Green's function, $1 / r$, maintains the same functional form on scaling the distance from the origin, $r$, only a subset of the full moment space is required for describing solutions to Poisson's equation from sources at the origin. That subspace is equivalent to the set of unique polynomials on a sphere of any radius. Using this constraint $\left(x^{2}+y^{2}+z^{2}=R^{2}\right)$ reduces the $\left(\begin{array}{c}n+2 \\ n\end{array}\right)$ homogeneous polynomials of degree $n$ to only $2 n+1$ linearly independent polynomials.

\section{Representation of Polytensor Space}

Analogous to Dette and Studden 44, define the $p$-tuple of moments for a given distribution as

$$
\mathbf{c}_{p}(\sigma)=\left(m_{0}(r ; \sigma), m_{1}(r ; \sigma), \ldots, m_{p-1}(r ; \sigma)\right)
$$

where

$$
m_{n}(r ; \sigma) \equiv \int_{S}(r \cdot \hat{x})^{n} \sigma(x) d^{2} \hat{x},
$$

are the moments of the distribution $\sigma$, integrated over $S$, the surface of the unit sphere. Since each individual moment, $m_{n}$ is a polynomial in $r$ corresponding to a tensor 
of order $n, \mathbf{c}_{p}$ corresponds to a polytensor. [14] The set of moments generated by a point mass at $\hat{r}_{i}$ is,

$$
\mathbf{c}_{p}\left(\hat{r}_{i}\right) \equiv\left(\left(r \cdot \hat{r}_{i}\right)^{0},\left(r \cdot \hat{r}_{i}\right)^{1}, \ldots,\left(r \cdot \hat{r}_{i}\right)^{p-1}\right) .
$$

The $p$-th spherical moment space is given by, $\mathbf{c}_{p}(\sigma)$, and represents the set of all polytensors up to order $p-1$ that can be expressed as integrals of $r^{(n)}$ over a signed measure on the sphere (as in Eq. B6). The space is convex and of dimension $p^{2}$. For positive measures, it can be investigated using the techniques of Ref. 44]. For signed measures, it is isomorphic to the $p^{2}$ dimensional vector space, $\mathbb{R}^{p^{2}}$. Further, using the reproducing kernel (Eq. 10), it is easy to see that every spherical polytensor $c_{p}$ admits a representation as a weighted sum over polytensors generated by fixed unit vectors,

$$
\mathbf{c}_{p}=\sum_{i=1}^{N} q_{i} \mathbf{c}_{p}\left(\hat{r}_{i}\right)
$$

where $N \leq p^{2}$.

This representation theorem shows that rather than the spherical harmonic coefficients usually used to describe moments, we can use a set of $O\left(p^{2}\right)$ weights, $w_{i}^{\sigma}=\sigma\left(\hat{r}_{i}\right) w_{i}^{0}$ associated to unit vectors, $\left\{\hat{r}_{i}\right\}$ in real space to represent Cartesian polytensors up to order $p-1$. Every operation on the Cartesian tensor can be translated to an equivalent operation using the polynomial representation of Eq. B8. In particular, any $n$-th order trace-free multipole tensor can be expressed in terms of only $\sim \frac{3}{2} p^{2}$ quadrature points,

$$
M^{(n)}=\sum_{i=1}^{N} q_{i} \hat{r}_{i}^{(n)} .
$$

The natural connection between polynomials and tensors developed in the previous section simplifies the use of this set for function expansions.

In summary, there is a direct correspondence between supersymmetric tensors and polynomials. This connection is usually used to generate reduced representations of supersymmetric tensors in terms of coefficients, $M\left[n_{1}, n_{2}, n_{3}\right]$, on basis monomials, $r_{x}^{n_{1}} r_{y}^{n_{2}} r_{z}^{n_{3}}$. 4, , 7, 11, 14] This representation is cumbersome because it requires a particular choice for the orientation of the coordinate axes, and (for scale-free problems) because it is not specialized for trace-tree polynomials. Using Eq. 12 and Eq. B9, quadrature rules for integrating polynomial functions give the desired compact and geometrically useful representation in terms of values taken by $m_{n}\left(r_{i}\right)$ or $\rho\left(r_{i}\right)$ at a small set of points, $r_{i}$, in real space. The basis vectors, $\left\{\hat{r}_{i}\right\}$, are much more computationally useful because they can be individually rotated / translated and their coefficients can be directly multiplied without re-factoring between monomials or computing ClebschGordon coefficients.
[1] G. G. Ferenczy, P. J. Winn, and C. A. Reynolds, J. Phys. Chem. A 101, 5446 (1997) http://pubs.acs.org/doi/pdf/10.1021/jp9712011.

[2] D. T. Chang, G. K. Schenter, and B. C. Garrett, J. Chem. Phys. 128, 164111 (2008)

[3] G. Beylkin and T. S. Haut, Proceedings of the Royal Society A: Mathematical, Physical and Engineering Science 469 (2013), 10.1098/rspa.2013.0231, http://rspa.royalsocietypublishing.org/content/469/2158/20

[4] A. J. Bordner and G. A. Huber, J. Comput. Chem. 24, 353 (2003).

[5] M. J. Schnieders, N. A. Baker, P. Ren, and J. W. Ponder, J. Chem. Phys. 126, 124114 (2007).

[6] M. J. Schnieders and J. W. Ponder, J. Chem. Theory Comput. 3, 2083 (2007)

[7] G. Dassios and M. Hadjinicolaou, Int. J. Eng. Sci. 40, 223 (2002).

[8] F. A. Cruz, M. G. Knepley, and L. A. Barba, Int. J. Numerical Methods Eng. 85, 403 (2011)

[9] A. Stone, Intermolecular Forces (Cambridge University Press, 1990).

[10] B. Jeziorski, R. Moszynski, and K. Szalewicz, Chemical Reviews 94, 1887 (1994), http://pubs.acs.org/doi/pdf/10.1021/cr00031a008.

[11] P. Ren and J. W. Ponder, J. Phys. Chem. B 107, 5933 (2003)

[12] C. Kramer, T. Bereau, A. Spinn, K. R. Liedl, P. Gedeck, and M. Meuwly, J. Chem. Inf. Model. 53, 3410 (2013).
[13] R. Anandakrishnan, C. Baker, S. Izadi, and A. V. Onufriev, PLoS One 8, e67715 (2013)

[14] J. Applequist, J. Phys. A: Math. Gen. 22, 4303 (1989).

[15] E. Cancès, B. Mennucci, and J. Tomasi, J. Chem. Phys. 107, $3032(1997)$

[16] J. P. Bardhan and M. G. Knepley, J. Chem. Phys. 135, 124107 (2011), 10.1063/1.3641485.

[17] J. P. Bardhan and M. G. Knepley, Comput. Sci. Disc. 5,

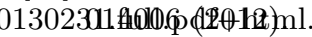

[18] A. Sawaryn and W. A. Sokalski, Comp. Phys. Comm. 52, 397 (1989)

[19] C. Pozrikidis, Boundary Integral and Singularity Methods for Linearized Viscous Flow (Cambridge Univ. Press, New York, 1992) p. 256.

[20] S. S. Turzi, J. Math. Phys. 52, 053517 (2011).

[21] K. Lorenzen, M. Schwörer, P. Tröster, S. Mates, and P. Tavan, J. Chem. Theory Comput. 8, 3628 (2012)

[22] M. Valiev, E. J. Bylaska, N. Govind, K. Kowalski, T. P. Straatsma, H. J. J. van Dam, D. Wang, J. Nieplocha, E. Apra, T. L. Windus, and W. A. de Jong, Comput. Phys. Commun. 181, 1477 (2010).

[23] A. Toukmaji, C. Sagui, J. A. Board, and T. Darden, J. Chem. Phys. 113, 10913 (2000).

[24] P. Ren and J. W. Ponder, Journal of Computational Chemistry 23, 1497 (2002)

[25] D. A. Case, T. E. Cheatham III, T. Darden, H. Gohlke, R. Luo, K. M. Merz, Jr., A. Onufriev, C. Simmerling, B. Wang, and R. Woods, J. Computat. Chem. 26, 1668 
(2005).

[26] L. D. Jacobson, C. F. Williams, and J. M. Herbert, J. Chem. Phys. 130, 124115:118 (2009)

[27] C. A. White and M. Head-Gordon, J. Chem. Phys. 101 (1994).

[28] C. Ahrens and G. Beylkin, Proceedings of the Royal Society A: Mathematical, Physical and Engineering Science 465, 3103 (2009) http://rspa.royalsocietypublishing.org/content/465/2110/310

[29] V. Lebedev, Siberian Math. J. 18, 99 (1977)

[30] J. Makino, J. Comp. Phys. 151, 910 (1999)

[31] L. Ying, G. Biros, and D. Zorin, J. Comp. Phys. 196, 591 (2004).

[32] Y. J. Liu and N. Nishimura, Eng. Analysis with Bound. Elem. 30, 371 (2006).

[33] C. Lessig, T. de Witt, and E. Fiume, J. Comp. Phys. 231, $243(2012)$

[34] J. Burkardt, "Quadrature rules for the sphere," http://people.sc.fsu.edu/\%7Ejburkardt (2010).

[35] A. Haigh, Implementation of rotation-based operators for
Fast Multipole Method in X10 (Austrailian National University, 2011).

[36] E. Darve, Journal of Computational Physics 160, 195 (2000)

[37] M. G. Reuter, M. A. Ratner, and T. Seideman, J. Chem. Phys. 131, 094108 (2009)

[38] M. Tygert, J. Comput. Phys. 227, 4260 (2008).

[39] C. de Boor and A. Ron, Math. Comp. 58, 705 (1992).

1029fjuS.pdffgthenlJ. Johnson, D. Padua, M. Püschel, and J. Xiong, ACM SIGSAM Bulletin Communications in Computer Algebra 35, 1 (2001).

[41] L. Greengard and V. Rokhlin, Acta Numerica 6, 229 (1997).

[42] E. Jones, T. Oliphant, P. Peterson, et al., "SciPy: Open source scientific tools for Python," (2001).

[43] M. Gasca and T. Sauer, Adv. Comput. Math. 12, 377 (2000).

[44] H. Dette and W. J. Studden, The Theory of Canonical Moments with Applications in Statistics, Probability, and Analysis (Wiley, New York, 1997) p. 327. 